\title{
Suicide Attempt in Elderly: An Overlooked Complication of Late-Life Depression
}

\author{
Yhan Batista $^{1}$, Novira Widajanti ${ }^{2} \cdot(\mathbb{D})$, Agustina Konginan ${ }^{3}(\mathbb{D})$ \\ ${ }^{1}$ Department of Internal Medicine, Faculty of Medicine, Universitas Airlangga - Dr. Soetomo General Hospital Surabaya, \\ Indonesia \\ ${ }^{2}$ Division of Geriatrics, Department of Internal Medicine, Universitas Airlangga - Dr. Soetomo General Hospital Surabaya, \\ Indonesia \\ ${ }^{3}$ Department of Psychiatry, Universitas Airlangga - Dr. Soetomo General Hospital Surabaya, Indonesia
}

\begin{abstract}
A B S T R A C T
Suicide attempt in the elderly is underestimated and underreported due to cultural reference. Triggering factors may result from stressful life events or acquired late-life depression. An elderly woman, 69-year-old, was rushed to the emergency room due to household cleaning liquid ingestion. There was a history of previous suicide attempts and severe depressive episodes. Since there was no sign of extreme intoxication, the patient was treated symptomatically. Comprehensive geriatric and psychiatric assessments concluded that the patient was in a depressive state. The patient was discharged with symptomatic treatment and antidepressants, but she completed the course two weeks later by drowning herself in a river.
\end{abstract}

Keywords: Suicide, Elderly, Chemical ingestion, Depression

Correspondence: Novira Widajanti

E-mail: novirawidajanti@yahoo.com

Article history: $\bullet$ Received 17 October $2021 \bullet$ Received in revised form 21 November $2021 \bullet$ Accepted 3 December $2021 \bullet$ Available online 31 January 2022

\section{INTRODUCTION}

Late-life depression is a major depressive disorder in adults 60 years of age or older. The major depressive disorder occurs in up to $5 \%$ of community-dwelling older adults, and 8 to $16 \%$ of older adults have clinically significant depressive symptoms. The numbers of major depressive disorders rise with increasing medical morbidity, with reported rates of 5 to $10 \%$ in primary care and as high as $37 \%$ after critical care hospitalizations (Taylor, 2014). Despite the availability of relatively efficacious treatments, geriatric depression continues to be underdiagnosed. The failure to recognize geriatric depression stems from the clinical complexity of the syndrome, overlapping symptoms with medical comorbidities, social bias, and barriers of the settings in which most depressed elderly patients are treated (Gatchel et al., 2017).

Suicidal behavior in older adults is a significant public health issue in many countries. It concerned specific risk factors for suicidal thoughts and behaviors among older people: psychiatric and neurocognitive disorders, social exclusion, bereavement, cognitive impairment, decision making, cognitive inhibition, physical illnesses, and physical and psychological pain (Conejero et al., 2018). Older adults experienced the final phase of life as a burden. They seemed to accept death, hence gradually developed suicidal ideation after a life full of adversity and due to aging, illness, or recurring depression (Holm et al., 2014).

This case aims to learn that suicide attempt in the elderly is an iceberg phenomenon in the community. It should not be underestimated since it may be a medical problem that requires comprehensive and extensive multidisciplinary approaches.

\section{CASE REPORT}

Mrs. L, 69-year-old, a widow, was brought by her son to the emergency room after ingesting household insecticide liquid 12 hours before admission. She complained of nausea and vomiting after ingesting approximately 250 milliliters of the liquid 12 hours before admission. She vomited 3-4 times with food contents and was accompanied by abdominal pain and soft stool defecation once. There was no trouble in swallowing meal or liquid. Excessive salivation, cough, lightheadedness, and seizure were denied. There was no problem with urinating. She intentionally ingested the household cleaning liquid to end her life. In a month, there was a decreasing appetite and a weight loss of 3 kilograms in one month. For one year, there was a history of diabetes mellitus controlled with metformin 500 milligrams three times daily. She had controlled hypertension for two years with amlodipine 10 milligrams daily. There was a history of being admitted to the Psychiatric ward one month ago for four days, diagnosed with severe depression episode and suicide attempt. She received clobazam 5 milligrams twice daily and sertraline 25 milligrams once daily. The history of recreational drugs abuse was denied. There is no psychosis history in her family. She is a widow with four children. Her husband, a former military, died 12 years ago due to a heart attack. She just moved into a second child's house for one month. Before moving into her second 
child's house, she lived in her third child's home for 15 years. In her second child's house, she felt depressed due to the poor environment. She complained that the water was dirty, the weather was hot and humid, and the house was small and uncomfortable though she felt her son and the family treated her well. She felt unhappy for most of her life because her husband had cheated on her, and her children did not live the way she expected. One month ago, she attempted to drown herself in a swamp following the voice in her head, but his son rescued her and rushed her to hospital, then admitted to the Psychiatry ward for four days. She frequently had terrible dreams, most vivid and only hearing male voices. After receiving psychiatric treatment, she did not hear any voices again. She is independent in her daily activities. While living at her third child's house, she was socially active, like joining group religious recitation, but she spent her time mostly inside the house in her second child's house.

From the physical examination, it was found weak condition with GCS 456. Blood pressure was 130/80 $\mathrm{mmHg}$; respiratory rate was 20 times per minute; heart rate was 88 beats per minute and axillary temperature $36,8^{\circ}$ Celsius. No miosis pupils and hypersalivation were found from head and neck examination. No abnormality was found in the chest examination. Epigastric tenderness was found from the abdominal examination - no abnormality was found in the extremities examination.

Laboratory results showed within normal range. Chest x-ray showed no abnormality with a cardiothoracic ratio of $54 \%$, and electrocardiography showed normal sinus rhythm with 92 beats per minute. The patient was consulted to Psychiatry Department in Emergency Room and assessed severe depressive episodes without psychotic symptoms. Advises were delaying psycho-pharmacy with close observation on organic conditions, placing at the near nurse station, putting away dangerous substances or tools that patients could use as suicide media and psychotherapy intervention for patients and family.

The patient was assessed chemical ingestion, suicide attempt, a severe depressive episode without psychotic symptoms, diabetes mellitus, and hypertension based on history taking, physical examination, and laboratory findings. Diagnostic plans were fasting blood glucose, 2-hour postprandial glucose, and HbA1c. Early management was intravenous line insertion with Ringer Dextrose 5\% 1000 milliliters per day and intravenous omeprazole 40 milligrams twice daily. Monitor plans were vital signs, complaints, and suicidal ideation.

On the second day of admission, the patient felt nauseous, decreased, vomiting decreased, and abdominal pain decreased. She felt regretful about what her doing. She had planned to drink the cleaning liquid because she thought she was a burden. Vital signs were stable. No abnormalities were found in the physical examination. A comprehensive geriatric assessment was performed. It showed mild dependency, no delirium, in risk of malnourishment, mild cognitive impairment, severe depressive state, no incontinence, low risk of venous thromboembolism, low risk of the decubitus ulcer, and the initial type of insomnia. Psychiatric evaluation results were positive eye contact, verbal communication was minimal and stuttered, consciousness was alert, time and place orientation was normal, the mood was depressive, thoughts processes were realistic and coherent with a preoccupation of her complaints, the perception was normal, intention and psychomotorwere diminished. Themultiaxial diagnosiswas:
Axis I: Severe depressive episode without psychotic symptoms and suicide attempt

Axis II: Schizotypal personality

Axis III: Chemical substance ingestion, controlled

diabetes mellitus, and hypertension

Axis IV: Problems with family support

Axis V: Global Assessment of Functioning Scale 50 - 41.

The therapy plan was B1 diet 2100 calories per day, $\mathrm{NaCl}$ 0,9\% infusion 1000 milliliter per day, intravenous omeprazole 40 milligrams twice daily, amlodipine 10 milligrams peroral once daily, sertraline 25 milligrams peroral once daily, clobazam 5 milligrams peroral daily, aripiprazole 5 milligrams peroral daily, family psychoeducation, supportive psychotherapy, close observation and setting harmful substances or tools away which patient could use as suicide media.

On the third day of admission, the patient felt no nausea, no vomiting, and abdominal pain. She could sleep soundly in the night. She still felt regret for what her doing. Vital signs were stable. Fasting blood glucose was $95 \mathrm{mg} / \mathrm{dL}, 2$-hour postprandial glucose was $123 \mathrm{mg} / \mathrm{dL}$ and HbAlc 6,2\%. Therapy continued as the previous day. On the fourth day of admission, the patient had no complaints. She wanted to be discharged. Vital signs were stable. A psychiatric evaluation was eye contact positive with no stutters. The mood was dysphoric, and preoccupation with being discharged. Psychiatry Department wanted to admit the patient, but she and her family refused further hospital stay. The patient was discharged with amlodipine 5-milligram peroral daily omeprazole 20 milligrams peroral daily, sertraline 25 milligrams peroral daily, clobazam 5 milligrams peroral daily, and aripiprazole 5 milligrams daily. The patient was expected to visit Psychiatry and Internal Medicine clinics three days afterward. Three days after being discharged, the patient visited the Internal Medicine clinic. She complained of feeling a little nauseous. She regretted the attempt and promised herself not to do it again. She would stay at her fourth child's house and planned to have activities to forget her doing. Vital signs were stable, and no abnormality was found in physical examination. A comprehensive Geriatric Assessment was performed, and it showed improvement only in insomnia screening and still in a depressive state. She then was prescribed domperidone 10 milligrams peroral three times daily, omeprazole 20 milligrams peroral once daily, and sucralfate syrup 10 milliliters peroral three times daily. She also visited the Psychiatry clinic and received sertraline 25 milligrams peroral daily, clobazam 5 milligrams peroral daily, aripiprazole 5 milligrams daily, and supportive psychoeducation, which is educating patient to talk about thoughts and feeling to the family, keeping harmful substances or tools away from the patient, close monitoring, medication compliance, educating patient's family to support the patient morally and mandatory return to Psychiatry Clinic. Still, two weeks later, the patient's child reported that the patient died completing a suicide attempt due to drowning in the river near the house.

\section{DISCUSSION}

The etiology of late-life depression is multifactorial, including biological, psychological, and social factors (Allan and Ebmeier, 2013). Some hypotheses are proposed, including the late-onset hypothesis, depression-executive dysfunction hypothesis, inflammation hypothesis, and vascular depression hypothesis (Gatchel et al., 2017). Predisposing risk factors for late-life depression include female, widowed or divorced status, history of previous 
depression, brain changes due to vascular problems, major physical and chronic disabling illnesses, polypharmacy, excessive alcohol use, social disadvantage and low social support, caregiving responsibilities for a person with a major disease and personality type. Precipitating risk factors for depression should also be considered, including recent bereavement, move from home to another place, adverse life events, chronic stress, social isolation, and persistent sleep difficulties (Wiese, 2011). Late-life depression can be classified into major depressive disorder, minor depressive disorder, dysthymic disorder, bipolar type I disorder, and adjustment disorder with depressed mood. Geriatric depression syndromes could be related to a medical condition and substance-induced depression (Alexopoulos, 2005).

A comprehensive approach to diagnosing late-life depression must be made. A careful history and mental state examination must be performed since depression is a clinical diagnosis. Cognition status must be obtained since cognitive impairment can be associated with LLD and may be due to the underlying depression or represent a comorbid neurocognitive disorder. Functional assessment is essential in the evaluation of older adults. It allows clinicians to determine an individual's ability to live independently and attend to daily needs. Assessment for suicidality must be obtained since it is an integral part of all psychiatric evaluations. Suicidality can range from passive thoughts of death and wishing that one were not alive to active thoughts of self-harm with plan and intent. Physical examination and ancillary studies are needed to identify underlying medical conditions possibly contributing to or mimicking depressive symptoms. Routine laboratory studies include complete blood count, complete metabolic panel, thyroid studies, and urine drug screen. Signs and symptoms of underlying medical conditions may necessitate further laboratory studies. Neuroimaging may reveal signs of cerebrovascular disease, which can predispose, precipitate, or perpetuate depression in older adults (Glover \& Srinivasan, 2017).

Clinical features of depression in elderlies may be different than in early ages. Some features specific to elderlies may make diagnosis and treatment of depression difficult. Elderlies have difficulty in expressing their depressive moods. Depressive moods, loss of interest, and anhedonia can be seen as a feature of elderliness. Vegetative symptoms such as sleeplessness, loss of appetite, and somatic symptoms such as constipation and pain are expressed more easily.

Along with aging, the frequency of medical diseases increases. The symptoms can be evaluated as part of the medical illnesses. When the losses and medical conditions that occur in elderlies are taken into account and assuming the depression symptoms as normal or acceptable, attributing tiredness, loss of appetite, and sleep disorders to medical diseases, the trend of hiding complaints if cognitive problems are encountered, difficulties in elderlies in expressing their emotions and their trend towards the somatization of their emotions negatively affect the diagnosis of depression. Hypochondriac complaints, psychomotor retardation, agitation, and psychotic symptoms in elderlies are more frequently found. Thought disorders may be found, such as delusions of persecution, guilt, and nihilism. Loss of attention and concentration and disorders in cognitive functions such as short-term memory and recall process can be observed (Sozeri-Varma, 2012).

Management of depression in the elderly consists of complex non-pharmacological and pharmacological interventions. Non-pharmacological therapy consists of lifestyle changes, e.g., increasing physical activities, improving nutrition, and increasing pleasurable activities and social interactions (Solomon, 2014). When selecting an antidepressant, it is important to consider the elderly patient's previous response to treatment, the type of depression, other medical problems, other medications, and the potential risk of overdose (Wiese, 2011).

Selective serotonin-reuptake inhibitors (SSRIs) are the first-line treatments for elder depression due to their favorable adverse-event profiles. Common adverse effects of SSRIs, typically mild, include nausea and headache. Serotonin-norepinephrine reuptake inhibitors (SNRIs) are commonly used as second-line agents when remission is not obtained with SSRIs. Tricyclic antidepressants have similar efficacy to SSRIs in treating late-life depression but are less commonly used owing to their greater side effects. If SSRIs or SNRIs are ineffective, tricyclic antidepressants may be considered. Second-generation antipsychotic agents such as olanzapine and aripiprazole have been increasingly used to treat nonpsychotic depression. In open-label studies, $50 \%$ of depressed older adults who did not have a complete response to an antidepressant were reported to have a remission with aripiprazole augmentation. Akathisia was the most common side effect in this population, occurring in $17 \%$ of older patients (Allan \& Ebmeier, 2013; Solomon, 2014).

Psychotherapies are effective treatments for latelife depression and may be considered first-line therapy. Standardized psychotherapeutic approaches include a short-term treatment phase consisting of weekly visits over 8 to 12 weeks. Although other therapies may also be effective, the evidence base for short-term treatment is most substantial for the cognitive behavioral therapy and problem-solving therapy. Cognitive-behavioral therapy focuses on identifying and reframing negative, dysfunctional thoughts while participating in pleasurable and social activities. Problem-solving therapy focuses on developing skills to improve the ability to cope with life problems. Electroconvulsive therapy (ECT) is the most effective treatment for severely depressed patients, including elderly patients. Although antidepressant medication is first-line therapy, ECT should be considered in patients if they are suicidal, have not responded to antidepressant pharmacotherapy, have a deteriorating physical condition, or have a depression-related disability that threatens their ability to live independently. Common side effects include postictal confusion with both anterograde and retrograde amnesia; current administration techniques, such as unilateral electrode placement with a brief pulse, substantially reduce this risk. Cognitive symptoms typically resolve after the completion of ECT. Persons with cardiovascular or neurologic diseases are at increased risk for ECT-related memory problems. (Allan \& Ebmeier, 2013; Solomon, 2014). Transcranial magnetic stimulation is a newer treatment for depression that uses a focal electromagnetic field. This treatment does not require anesthesia and does not have cognitive side effects (Solomon, 2014).

Major depression is a risk factor for suicide, as is substance abuse. Mood disorders constitute an independent risk factor for suicide in elderly people, whereas physical illness and disability increase suicide risk, but their effect is mediated by depression. Disruption of social ties is associated with late-life suicide independently of 
depression, especially in individuals with rigid, anxious, and obsessive personalities. Availability of firearms doubles the risk of suicide in elderly people. Suicidal ideation decreases with aging, but if older people have suicidal thoughts, they are more likely to commit suicide than younger people. Suicidal ideation is strongly associated with the severity of depression. Findings of a study show that during an initial assessment of elderly patients with major depression, the severity of depression and previous serious attempts could predict the course of suicidal ideation. During follow-up, contemporaneous severity of depression was the most crucial determinant of suicidal ideation over time. These observations can be used to identify elderly people with depression who are at high risk of committing suicide (Alexopoulos, 2005). Identification, prevention, and treatment of depression are pivotal for preventing suicide in late life. The approach must be made by conducting an assessment of suicide risk, developing a cognitive case conceptualization, developing a safety plan, increasing hope and reasons for living, improving social resources, improving problem-solving skills and efficacy, improving adherence to medical regimen, and relapse prevention (Bhar \& Brown, 2012).

Risk factors must be identified to prevent suicide. Known risk factors are suicidal behavior, suicide ideation, mental illness, addiction, personality factors, medical illness, negative life events, transitions, social support variables, and functional impairment. Conversely, there are resiliency or protective factors for elder suicide that could reduce the risk of suicide as better health care practices, contact with family and friends, moderate alcohol consumption, active interests, religious practice, recognition of purposes of life, and personal attributes such as openness to experience, extraversion, and conscientiousness. Perceived meaning in life, life satisfaction, adaptive coping, future orientation, and spirituality might increase psychological wellbeing and decrease suicide risk. Measurement tools for assessing suicide risk still need further validation (CCSMH, 2006).

The effectiveness of psychiatric treatment across the diagnostic spectrum must be met to achieve suicide risk reduction. Pharmacological intervention for psychiatric illness, psychotherapy, electroconvulsive therapy, and hope enhancement should benefit. Interpersonal relationships between therapists and clients and support from a health care professional would give more advantages to decrease suicide risk (CCSMH, 2006). In this case, the difficulty in educating the patient's family about the importance of medication, close monitoring, social support, and the depth of depressive state that the patient experienced may contribute to the failure of management.

\section{SUMMARY}

A 69-year-old patient with a suicide attempt with severe depressive episodes without psychotic symptoms, diabetes mellitus, and hypertension has been reported. The patient was treated symptomatically since the intoxication was mild and reintroduced to antidepressants to improve compliance. Suicide attempts in the elderly must be taken seriously since they have a higher number of completions. Multidisciplinary approaches in suicide attempts of depressive elders must be considered since the complexities of elderly features blunt the symptoms of elderly depression. Early psychiatric consultation may improve the patient's outcome, and the importance of social support surrounding the patient plays a critical factor in determining which approach is best suited to the patient.

\section{CONFLICT OF INTEREST}

The authors declare there is no conflict of interest.

\section{REFERENCES}

Alexopoulos, GS. 2005. Depression in the elderly. Lancet. 365(1):1961 - 1970 .

Allan CL, Ebmeier KP. 2013. Review of treatment for late-life depression. Advances in Psychiatric Treatment 19(1):302-309.

Bhar SS, Brown, GK. 2012. Treatment of depression and suicide in older adults. Cognitive and Behavioral Practice 19(1):116-125.

Canadian Coalition for Seniors' Mental Health. 2006. National guidelines for seniors' mental health: the assessment of suicide risk and prevention of suicide, p 15-21. Available at https://ccsmh.ca/wp-content/ uploads/2016/03/NatlGuideline_Suicide.pdf.

Conejero I, Olié E, Courtet P, Calati R. 2018. Suicide in older adults: current perspectives. Clinical Interventions in Aging 13(1):691-699.

Gatchel JR, Wilkins JM, Forester BP, Kelly Jr. RE, Alexopoulos GS. 2017. Geriatric mood disorders. In BJ Sadock, VA Sadock and P Ruiz (Eds). Kaplan \& Sadock's Comprehensive Textbook of Psychiatry, 10th Edition. Philadelphia, Wolters Kluwer, p 10331 - 10371.

Glover JA, Srinivasan S. 2017. Assessment and treatment of late-life depression. Journal of Clinical Outcomes Management 24(3):1-13.

Holm AL, Lyberg A, Berggren I, Åström S, Severinsson E. 2014. Going around in a circle: a norwegian study of suicidal experiences in old age. Nursing Research and Practice 1(1):1-9.

Reynolds CF. 2017. Evidence-based treatment and prevention of major depressive episodes in later life. In: Halter, JB, Ouslander JG, Studenski S, High KP, Asthana $\mathrm{S}$, Ritchie CS, et al. (Eds). Hazzard's Geriatric and Gerontology Medicine, 7th edition. New York, McGrawHill, p 1294-1313.

Singh R, Mazi-Kotwal N, Thalitaya MD. 2015. Recognising and treating depression in the elderly. Psychiatria Danubina 27(1):231-234.

Sözeri-Varma G. 2012. Depression in the elderly: clinical features and risk factors. Aging and Disease 3(6):465-471. Taylor WD. 2014. Depression in the elderly. New England Journal of Medicine 371(1):1228-1236.

Wiese BS. 2011. Geriatric depression: the use of antidepressants in the elderly. British Columbia Medical Journal 53(7):341-347. 